\title{
Uncertain Program Based Unit Commitment Model for Power Grid Including Wind Farms
}

\author{
SHENG Siqing, ZHANG Li \\ School of Electrical and Electronic Engineering, North China Electric Power University, China \\ jsuzhl@163.com
}

Keywords: Wind farms; Uncertain programming; Forecast errors; hybrid chance-constrained model; Evolutionary algorithm

\begin{abstract}
Taking the multiple uncertainties commonly existing in electrical power networks including wind farms into consideration, this paper introduces the uncertain programming theory to convert the uncertainties of wind power and load to those of their forecast errors. Making utilization of fuzzy variable and random variable, the different characteristics of wind power and load are expressed respectively to establish a novel hybrid chance-constrained unit commitment model. In order to solve the established model, a hybrid-simulation evolutionary algorithm is proposed and is applied to the following simplified test system. The validity and effectiveness of the proposed hybrid chance-constrained unit commitment model are demonstrated by the simulation results and analysis.
\end{abstract}

\section{Introduction}

As a new kind of clean and renewable energy, wind energy has been vigorously developed all over the world. According to statistics [1], by the end of 2013, not only the newly-increased installed capacity but also the cumulative one occupy the first places in the world. Wind power will make greater contribution to China's economic development and utilization of environmental resources[2-3]. However, with the scale of grid-connected wind power becoming larger, wind power has also brought about a lot of adverse effects [4]. Therefore it is of vital importance to carrying out research in the impact of wind power integration on power system.

As the strong intermittent and volatility of wind power will add uncertainty to dynamic economic dispatch of power system [5-6], scholars at home and abroad have conducted extensive and deep researches on the economic dispatch problem for power system with wind power integration [7]. Literature [8] sets up a dynamic economic dispatch model via the theory of stochastic programming, considering wind power penetration. On the basis of wind speed forecasting, the paper combines intelligent algorithm, neural network and genetic algorithm to solve the mode. The hybrid algorithm is quite enlightening.

In this paper, taking common multiple uncertain factors of power system into account, a novel hybrid chance-constrained unit commitment model containing fuzzy variable and random variable at the same time is established through uncertain programming theory. In order to solve the established model, a hybrid-simulation evolutionary algorithm is put forward. Avoiding complex mathematical deduction, the algorithm possesses its own superiority as it is simple in principles and is clear in physical concept. In addition, the optimization results of two types of confidence levels demonstrate the validity and effectiveness of the hybrid chance-constrained unit commitment model established in this paper. Based on the results, the system dispatchers can select proper confidence level to satisfy the requirements of economical efficiency and operating reliability.

\section{Unit Commitment Model for Electrical Power Grid Including Wind Farms}

Objective function. The traditional form of objective function is used to solve the problem. Select the minimum of normal unit operating cost as objective function. The model is as follow: 


$$
\min C=\sum_{t=1}^{T} \sum_{i=1}^{N}\left[f\left(p_{i t}\right)+S_{i t} u_{i t}\left(1-u_{i(t-1)}\right)\right]
$$

where $C$ represents the total operating cost of thermal power units, including both operating cost and the unit start-up cost; $f\left(p_{i t}\right)$ represents the operating cost; $p_{i t}$ represents the output of the thermal power unit named as $i$ at moment $\mathrm{t}$; Sit is the start-up cost of thermal power unit $i$ at moment $t ; u_{i t}$ is the running state of thermal power unit $i$ at moment $t$, of which 0 represents shutdown and 1 represents startup; $\mathrm{T}$ is the whole scheduling period; $\mathrm{N}$ is the total number of thermal power units.

The operating cost can be expressed as:

$$
f\left(p_{i t}\right)=a_{i} p_{i t}^{2}+b_{i} p_{i t}+c_{i}
$$

where $a_{i}, b_{i}, c_{i}$ are represent the operating cost coefficients of the thermal power unit $i$, respectively. Constraint Conditions. Given the values of wind power prediction and load forecast, the constraint conditions of power balance can be expressed as:

$$
\sum_{i}^{N} p_{i t}+p_{w t y}=p_{l t y}
$$

where $p_{\text {imax }}$ is the predicted output of wind power at moment $\mathrm{t}$ and $p_{\text {Ity }}$ is the predicted output of load at moment $t$.

According to the actual condition of power system scheduling operation, we distribute predicted values to the load and keep spinning reserve for the prediction error. So the constraint condition of spinning reserve can be expressed as:

$$
\sum_{i=1}^{N} u_{i t} p_{i \max }+p_{w t y}+\xi_{w t} \geq p_{l t y}+\eta_{l t}
$$

where $p_{\text {imax }}$ is the maximum technical output of the thermal power unit $i$; $\xi_{w t}$ is the prediction error of wind power at moment $t ; \eta_{l t}$ is the prediction error of load at moment $t$.

Because wind power generation is affected by the environmental factors such as temperature and climate, the output of wind power often has the feature of fuzziness, so it can be expressed by a triangular fuzzy parameter. As the output of daily load is highly repetitive, it is generally expressed as random variables obeying the normal distribution. Specific parameter values can be obtained by the historical statistical data. Thus the originally certain inequality is converted to the uncertain one containing both fuzzy variables and random variables. We can introduce fuzzy and random confidence levels to obtain constraint conditions for spinning reserve:

$$
\operatorname{Pos}\left\{\operatorname{Pr}\left\{\sum_{i=1}^{N} u_{i t} p_{i \max }+p_{w t y}+\xi_{w t} \geq p_{l t y}+\eta_{l t}\right\} \geq \alpha\right\} \geq \beta
$$

where $\alpha$ is the confidence level for the random variable and $\beta$ is the confidence level for the fuzzy variable.

The upper limit and lower limit of thermal power unit output can be expressed as:

$$
p_{\text {imin }} u_{i t} \leq p_{i t} \leq p_{i \max } u_{i t}
$$

where $p_{i \max }$ is the maximum technical output of thermal power unit $i$ and $p_{i \min }$ is the minimum technical output of the thermal power unit $i$.

The climbing constraint of thermal power unit keeping start-up can be expressed as: 


$$
D_{i} \leq p_{i t}-p_{i(t-1)} \leq U_{i}
$$

where $D i$ is the down-climbing constraint of the thermal power unit $i$ and $U i$ is the up-climbing constraint of the thermal power unit $i$.

The minimum times of thermal power units startup and shutdown can be expressed as

$$
\begin{aligned}
& T_{i t o n} \geq T_{i \text { minon }} \\
& T_{\text {itoff }} \geq T_{\text {imin off }}
\end{aligned}
$$

where $T_{i t o n}$ is the start-up time of thermal power unit $i$ until the moment $t$; $T_{\text {itoff }}$ is the shut-down time of thermal power unit $i$ until the moment $t ; T_{i \text { min on }}$ is the allowed minimum startup time of thermal power $i$; $T_{i \text { min off }}$ is the allowed minimum shutdown time of thermal power unit $i$.

\section{Model Solving}

Considering the current situation that part of thermal power units must keep start-up for 24 hours due to the carrying base load, we can set these units as normally start-up unit to reduce the burden of random search. Since the operating efficiencies of the units are different from each other, in order to guarantee priority startup and operation of those high-efficiency units, this paper evaluates the operating efficiencies of units. Calculating formulas are as follows:

$$
\begin{aligned}
& L_{i}=\frac{a_{i} p_{i, a v g}^{2}+b_{i} p_{i, a v g}+c_{i}}{p_{i, a v g}} \\
& p_{i, a v g}=\left(p_{i \max }+p_{i \min }\right) / 2
\end{aligned}
$$

The concrete steps to run the algorithm are as follows:

a. Set the system parameters and initialize the population.

b. Test the constraint of spinning reserve. Use the hybrid simulation test strategy to determine whether each chromosome satisfies the spinning reserve constraint or not. If it satisfies, stop the redundant units according to the shutdown priority until it cannot reduce any unit but still meets the spinning reserve constraint. Otherwise, start the unit one by one according to the priority order of startup units to meet requirements of spinning reserve. Go to Step (c) when spinning reserve constraint is satisfied.

c. Test time constraints of startup and shutdown. Determine whether each chromosome satisfies the minimum-time constraints of units' startup and shutdown by the actual minimum times of units' startup and shutdown. If constraints are satisfied, enter Step (d); otherwise, modify the chromosome and test it by the constraint of spinning reserve. Return Step (b).

d. Calculate the fitness function value of each chromosome and update the optimal solution.

e. Use evolutionary operation to update the population. For the new chromosomal, repeat Steps (b) - (d).

f. Determine whether reaching the termination conditions or not. If it is, stop the process; otherwise return (e).

\section{Analysis of Examples}

According to the unit evaluation indexes in this paper, priority orders to star up the ten thermal power units are shown in Table 1.

Table 1. Priority orders to start up units.

\begin{tabular}{ccccccccccc}
\hline Order of units startup and shutdown & 1 & 2 & 3 & 4 & 5 & 6 & 7 & 8 & 9 & 10 \\
\hline Unit number & 1 & 2 & 5 & 4 & 3 & 6 & 7 & 8 & 9 & 10 \\
\hline
\end{tabular}


According to the model in this paper, when random confidence level is 0.95 and fuzzy confidence level is 0.3 , optimization results are shown in Table 2. The total operating cost in the whole scheduling period is as much as $5.81 \times 105$ dollars. In addition, according to the data in the Table 2 , the thermal power units start up as the priority orders in Table 1.

Table 2. Results of optimal scheduling(MW).

\begin{tabular}{ccccccccccc}
\hline Period number & Unit1 & Unit2 & Unit3 & Unit4 & Unit5 & Unit6 & Unit7 & Unit8 & Unit9 & Unit10 \\
\hline 1 & 366 & 154 & 0 & 0 & 0 & 0 & 0 & 0 & 0 & 0 \\
2 & 385 & 210 & 0 & 0 & 0 & 0 & 0 & 0 & 0 & 0 \\
3 & 444 & 311 & 0 & 0 & 0 & 0 & 0 & 0 & 0 & 0 \\
4 & 452 & 279 & 0 & 0 & 94 & 0 & 0 & 0 & 0 & 0 \\
5 & 450 & 331 & 0 & 0 & 44 & 0 & 0 & 0 & 0 & 0 \\
6 & 452 & 330 & 0 & 113 & 55 & 0 & 0 & 0 & 0 & 0 \\
7 & 446 & 397 & 0 & 108 & 59 & 0 & 0 & 0 & 0 & 0 \\
8 & 441 & 376 & 87 & 120 & 81 & 0 & 0 & 0 & 0 & 0 \\
9 & 454 & 446 & 118 & 115 & 127 & 0 & 0 & 0 & 0 & 0 \\
10 & 449 & 432 & 128 & 123 & 134 & 61 & 53 & 0 & 0 & 0 \\
11 & 451 & 431 & 128 & 98 & 135 & 72 & 49 & 0 & 0 & 0 \\
12 & 454 & 419 & 125 & 123 & 123 & 42 & 77 & 43 & 0 & 0 \\
13 & 453 & 447 & 117 & 124 & 149 & 39 & 0 & 0 & 0 & 0 \\
14 & 454 & 447 & 88 & 127 & 74 & 0 & 0 & 0 & 0 & 0 \\
15 & 450 & 365 & 116 & 117 & 27 & 0 & 0 & 0 & 0 & 0 \\
16 & 336 & 267 & 121 & 119 & 27 & 0 & 0 & 0 & 0 & 0 \\
17 & 312 & 273 & 113 & 90 & 27 & 0 & 0 & 0 & 0 & 0 \\
18 & 432 & 229 & 100 & 71 & 59 & 0 & 0 & 0 & 0 & 0 \\
19 & 451 & 326 & 121 & 99 & 38 & 0 & 0 & 0 & 0 & 0 \\
20 & 454 & 431 & 91 & 118 & 67 & 0 & 0 & 40 & 0 & 0 \\
21 & 453 & 422 & 117 & 126 & 67 & 0 & 0 & 0 & 0 & 0 \\
22 & 447 & 404 & 0 & 112 & 60 & 0 & 0 & 0 & 0 & 0 \\
23 & 449 & 431 & 0 & 0 & 0 & 0 & 0 & 0 & 0 & 0 \\
24 & 380 & 340 & 0 & 0 & 0 & 0 & 0 & 0 & 0 & 0 \\
\hline
\end{tabular}

Fix the value of fuzzy confidence level as 0.3. Unit startup and shutdown states and comparison of 10 thermal power units start-up costs under different random confidence levels are listed in Table 3.

Table 3. Unit startup and shutdown states and comparison of startup costs under different random confidence level.

\begin{tabular}{|c|c|c|c|c|c|c|c|c|c|}
\hline \multirow{2}{*}{ Period } & $\mathrm{A}^{*}=0.8$ & & $A^{*}=0.95$ & Cost/\$ & \multirow{2}{*}{ Period } & \multirow{2}{*}{$\begin{array}{c}\mathrm{A}^{*}=0.8 \\
\text { Unit states }\end{array}$} & \multirow{2}{*}{ Cost $/ \$$} & $A^{*}=0.95$ & \multirow{2}{*}{ Cost $/ \$$} \\
\hline & Unit states & & Unit states & & & & & Unit states & \\
\hline 1 & 1100000000 & 0 & 1100000000 & 0 & 13 & 1111101000 & 0 & 1111110000 & 0 \\
\hline 2 & 1100000000 & 0 & 1100000000 & 0 & 14 & 1111100000 & 0 & 1111100000 & 0 \\
\hline 3 & 1100000000 & 0 & 1100000000 & 0 & 15 & 1111100000 & 0 & 1111100000 & 0 \\
\hline 4 & 1100100000 & 1800 & 1100100000 & 1800 & 16 & 1111100000 & 0 & 1111100000 & 0 \\
\hline 5 & 1100100000 & 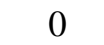 & 1100100000 & 0 & 17 & 1111100000 & 0 & 1111100000 & 0 \\
\hline 6 & 1100100000 & 0 & 1101100000 & 1120 & 18 & 1111100000 & 0 & 1111100000 & 0 \\
\hline 7 & 1101100000 & 1120 & 1101100000 & 0 & 19 & 1111100000 & $\sigma$ & 1111100000 & 0 \\
\hline 8 & 1101100000 & 0 & 1111100000 & 1100 & 20 & 1111100000 & 60 & 1111100100 & 60 \\
\hline 9 & 1111100000 & 1100 & 1111100000 & 0 & 21 & 1111100000 & 0 & 1111100000 & 0 \\
\hline 10 & 1111110000 & 340 & 1111111000 & 860 & 22 & 1111000000 & 0 & 1101100000 & 0 \\
\hline 11 & 1111111000 & 520 & 1111111000 & 0 & 23 & 1100000000 & 0 & 1100000000 & 0 \\
\hline 12 & 1111111000 & 0 & 1111111100 & 60 & 24 & 1100000000 & 0 & 1100000000 & 0 \\
\hline
\end{tabular}

* A represents random confidence level.

When random confidence level is 0.8 or 0.95 , the spinning reserve is 5001 or 5428 megawatts during the whole scheduling period. Analysis of the data above shows that when the fuzzy confidence level is constant, if the random confidence level is improved, the thermal power units will start up in advance, as unit 8 start up earlier under the random confidence level 0.95 to satisfy the system constraints. Random confidence level reflects the reliability of system energy supply. The higher the random confidence level is and the more adequate the spinning reserve is, the higher total cost will be. The scheduling decision makers can set different random confidence levels according to the actual 
situation of system. Fix the random confidence level as 0.95 . The comparison of optimization results under different fuzzy confidence levels are shown in Table 4.

Table 4. The optimization results under different fuzzy confidence levels (\$).

\begin{tabular}{cccc}
\hline$B^{*}$ & C $^{*}$ & $\mathrm{D}^{*}$ & $\mathrm{E}^{*}$ \\
\hline 0.3 & 5000 & $5.81 \times 105$ & 5428 \\
0.6 & 4480 & $5.79 \times 105$ & 4359 \\
0.9 & 4480 & $5.78 \times 105$ & 3374
\end{tabular}

* B represents fuzzy confidence level. C represents start-up cost. D represents total operation cost. E represents spinning reserve capacity.

From analysis of the data in Table 4, with the improvement of fuzzy confidence level, the start-up costs reduce greatly and the total operating costs also reduced. Because of decreasing in the start-up number of units, spinning reserve capacity is reduced. Fuzzy confidence level reflects the reliability of wind power prediction. If fuzzy confidence level is low, the credibility of wind forecast is low, so more thermal power units should be start up in response to the lack of wind power. At this time, the start-up cost as well as the total operating cost is very high. If we use deterministic modeling methods, namely, reserve the total wind power and reserve $10 \%$ of the predicted load, the spinning reserve capacity will be as much as 5605 megawatts and start-up cost will reach 5980 dollars. Compared with the uncertain model built in this paper, the controllability of the whole system will be abated and the requirement of reserve capacity will be greater. Thus, to realize the system economical and reliable operation, dispatchers can set reasonable confidence level based on the actual situation of system.

\section{Conclusion}

Taking the multiple uncertain factors commonly existing in power system into account, a novel hybrid chance-constraint unit commitment model containing the uncertainties of both wind power and load is established, in which the uncertainties of wind power and load output are replaced by different types of predicted errors. Example analysis proves the feasibility of the model and the advantages in improving system controllability. Moreover, the analysis in this paper makes it clear for power dispatchers to recognize the relationship between risk and benefits, providing a new idea for scheduling rules.

\section{References}

[1] Chinese Wind Energy Association. 2014. China's installed wind capacity in 2013. Wind Energy 2: 44-55.

[2] X.T. Dong, Z. Yan, D.H. Feng, et al: Modern Electric Power Vol. 29(2012), p. 42-48.

[3] X.H. Wang, L.Z. Zhang, S.J. Cheng, et al: Modern Electric Power Vol. 31(2014), p. 1-6.

[4] B.Q. Ren, C.W. Jiang, M.H. Peng, et al: Modern Electric Power Vol. 27(2010), p. 76-81.

[5] Y.W. Jiang, C. Chen, B.Y.Wen: Transactions of China Electrotechnical Society Vol. 24(2009), p. 129-137.

[6] N.Y. Zhang, S. Gao, X. Zhao: Transactions of China Electrotechnical Society Vol. 28(2013), p. 22-29.

[7] H.Y. Chen, J.F. Chen, X.Z. Duan: Automation of electric power systems Vol. 30(2006), p. 22-26.

[8] Y.Z. Sun, J. Wu, G.J. Li, et al: Proceedings of the CSEE Vol. 29(2009), p. 41-47.

[9] H. Xiong, T.Y. Xiang, H.K. Chen, et al: Proceedings of the CSEE Vol. 33(2003), p. 36-44. 